\title{
Obituaries
}

\section{Professor Tsung-yi Lin}

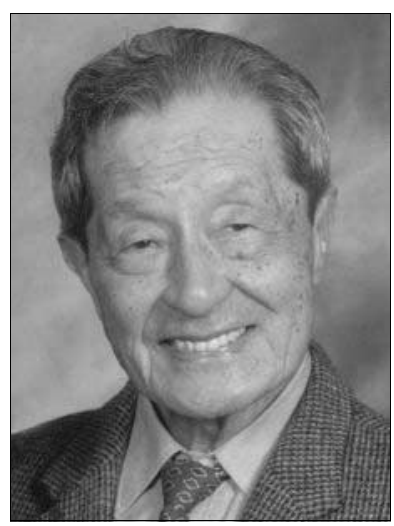

Formerly, inter alia, Professor of Psychiatry and Public Health, Michigan, USA

Professor Tsung-yi Lin was more than a world-famed psychiatrist: he was also a politician of international renown, particularly as regards the welfare of children. Born in 1920, to a family of scholars and educators, Tsung-yi Lin completed his training in medicine in Japan during the Second World War and graduated from the Tokyo Imperial University School of Medicine in 1943. Four years later, he founded the Department of Neuropsychiatry of the National Taiwan University in Taipei, developed plans for mental health services in Taiwan and initiated a series of epidemiological studies there. Subsequently, he continued his studies of psychiatry, first as a research fellow in the Harvard Medical School (1950-1952) and later as a World Health Organization (WHO) fellow at the Maudsley Hospital in London (1956-1957). His extraordinary success in building up a mental health service in Taiwan as well as his contributions to science were among the reasons that he was invited to lead the social and epidemiological psychiatry sector of the WHO Mental Health Unit in Geneva (1964-1969). During his time at the $\mathrm{WHO}$, he initiated several projects, including an international initiative to help in the development of diagnosis and classification of psychiatric disorders and the International Pilot Study of Schizophrenia, a major international collaborative project, supported by the National Institute for Mental Health in the USA and involving nine centres in different parts of the world.

Tsung-yi Lin left the WHO in 1969 and took the position of Professor of Psychiatry and Public Health in Michigan, USA. In 1973 he became Professor of Psychiatry at the University of British Columbia in Vancouver, Canada. He also taught as an honorary professor at the Tokyo University and at the Beijing
Medical University. He was an outstanding teacher and during his professorial life he has trained a number of future leaders of psychiatry. He was a member of the WHO Mental Health Expert Panel and served as a consultant to the $\mathrm{WHO}$ on a number of occasions, notably to help develop the undergraduate and postgraduate education in psychiatry in Asia. He published a number of articles and several books, including a volume on epidemiological psychiatry in the WHO Public Health series and, together with Professor Eisenberg, a book on mental health programmes in China - Mental Health Planning for a Billion People (1985). He served as the President of the World Federation for Mental Health and, during his presidency, strengthened the organisation and led it to new fields of work, including the powerful advocacy of the prevention of mental disorders. In honour of his major contributions to epidemiological psychiatry, he was elected an Honorary Member of the International Federation of Psychiatric Epidemiology (2007).

His achievements over the years have been truly remarkable. To build an excellent department of psychiatry in a low-income country would be a lifetime achievement for many of the best experts in the field of mental health - and that was only the beginning of Tsung-yi Lin's long and highly productive career. His contribution to the epidemiology of mental disorders - both by original scientific investigations and by giving epidemiology in psychiatry an international focus - were most remarkable, yet pale in comparison with his later humanitarian efforts in often very difficult circumstances.

He died at the age of 89 on 20 July 2010 . He is survived by his beloved and devoted wife, who shared his life for 65 years and their five children, of whom one, Elizabeth Lin, has already made her international mark by outstanding contributions to the field of general practice psychiatry, in the same spirit as her father. The four of us remember Tsung-yi Lin as a remarkable leader with great personal charm, charisma, vision and empathy for people in distress, whether because of mental illness or other difficulties.

Professor Norman Sartorius,

Emeritus Professor of Psychiatry John Cooper Professor Andrew T. A. Cheng, Professor Mian-Yoon Chong 